\title{
Brentuximab vedotin maintenance after autologous stem cell transplantation for refractory gray zone lymphoma with long-term remission
}

\author{
TOSHIKI TERAO ${ }^{1,2}$, JUNICHIRO YUDA ${ }^{1}$, NOBUHIKO YAMAUCHI ${ }^{1}$, YONG-MEI GUO $^{1}$, KAORU SHIMADA $^{3}$, \\ MASATO SUGANO ${ }^{4}$, GENICHIRO ISHII ${ }^{4}$ and YOSUKE MINAMI ${ }^{1}$ \\ ${ }^{1}$ Department of Hematology, National Cancer Center Hospital East, Kashiwa, Chiba 277-8577; \\ ${ }^{2}$ Division of Hematology/Oncology, Department of Internal Medicine, Kameda Medical Center, Kamogawa, Chiba 296-8604; \\ Departments of ${ }^{3}$ Radiology and ${ }^{4}$ Pathology, National Cancer Center Hospital East, Kashiwa, Chiba 277-8577, Japan
}

Received January 8, 2021; Accepted April 1, 2021

DOI: $10.3892 / \mathrm{mco} .2021 .2287$

\begin{abstract}
Gray zone lymphoma (GZL) is a rare type of B-cell lymphoma characterized by features of both diffuse large B-cell lymphoma and classical Hodgkin lymphoma (cHL). The prognosis of GZL is poorer than that of $\mathrm{cHL}$ and mediastinal large B-cell lymphoma. However, an optimal treatment strategy for relapsed/refractory (R/R) GZL has not been established in the clinical setting. The current study reported an excellent clinical response in a patient with R/R CD30-positive GZL who received brentuximab vedotin (BV) maintenance after autologous stem cell transplantation (ASCT). Although the patient was resistant to prior treatments, BV maintenance after ASCT achieved long-term remission. Hence, $\mathrm{BV}$ was determined to be a safe and effective therapeutic option for CD30-positive R/R GZL.
\end{abstract}

\section{Introduction}

Gray zone lymphoma (GZL) is a rare type of B-cell lymphoma, with features intermediate between diffuse large B-cell lymphoma (DLBCL) and classical Hodgkin lymphoma (cHL) (1). Despite advancements in immunophenotyping and molecular diagnostics in addition to the conventional morphologic approach, the diagnosis of GZL remains complex and challenging (2). Although GZL is commonly observed in young adults, the survival of patients with GZL is worse than that of patients with DLBCL or cHL. That is, one-third of patients present with primary refractory disease, and the 2-year progression-free survival (PFS) rate is $\sim 40 \%(3,4)$. Although its outcomes are inferior, an optimal treatment strategy for relapsed/refractory $(\mathrm{R} / \mathrm{R}) \mathrm{GZL}$ has not yet been identified.

Correspondence to: Dr Junichiro Yuda, Department of Hematology, National Cancer Center Hospital East, 6-5-1 Kashiwano-ha, Kashiwa, Chiba 277-8577, Japan

E-mail: jyuda@east.ncc.go.jp

Key words: gray zone lymphoma, relapsed, refractory, brentuximab vedotin, CD30
Allogenic stem cell transplantation (SCT) for R/R GZL may be a treatment option. However, the incidence of transplant-related mortality (TRM) or graft-versus-host disease is still a major problem. Therefore, more effective and less toxic therapy should be developed for patients with R/R GZL.

Brentuximab vedotin (BV), an anti-CD30 antibody drug conjugate, is highly effective for different types of lymphomas, including CD30-positive non-Hodgkin lymphoma and cHL (5-7). Recently, in the AETHERA trial, BV maintenance after autologous SCT (ASCT) had a statistically significant prognostic impact in patients with $\mathrm{R} / \mathrm{R} \mathrm{cHL}$ (8). However, only few studies showed that $\mathrm{BV}+$ conventional chemotherapy is effective for treatment-naïve GZL or BV maintenance therapy for R/R GZL $(5,9,10)$. Herein, we report a patient with R/R GZL that is highly resistant to conventional chemotherapy and radiation therapy (RT). The patient achieved long-term remission after receiving BV maintenance treatment after ASCT.

\section{Case report}

A 19-year-old male patient was referred to our institution for further examination of left neck and right axillary lymphadenopathy and anterior mediastinal mass without B symptoms. The patient's lactate dehydrogenase and soluble interleukin-2 receptor levels were high at $317 \mathrm{U} / 1$ (normal upper limit: $245 \mathrm{U} / \mathrm{l}$ ) and 2,040 U/ml, respectively. With consideration of malignant lymphoma, the patient underwent left neck lymph node biopsy. The pathological finding showed morphologic proliferation of atypical large lymphoma cells and the presence of few polynuclear Reed-Sternberg (RS)-like cells surrounded by infiltrating inflammatory cells (Fig. 1A). On immunohistochemical studies, the large lymphoma cells were positive for CD15, CD20 (Fig. 1C), CD30 (Fig. 1E), CD79a, PAX-5, and PD-L1 and were negative for CD5 and EBER. The Ki-67 index was 80\%. Collectively, after a cautious review, the patient was diagnosed with GZL. Positron emission tomography (PET)/computed tomography (CT) scan was performed prior to treatment. Results revealed that the maximum standardized uptake value (SUVmax) was 11.4 (Fig. 2A). After completing eight cycles of dose-adjusted etoposide, prednisone, vincristine, cyclophosphamide, doxorubicin, 
and rituximab (DA-EPOCH-R), PET/CT scan revealed a residual fluorodeoxyglucose (FDG)-uptake lesion, with an SUVmax of 6.8 , in the mediastinum. Lymph node biopsy was performed with a mediastinoscope to identify the pathological characteristics of the residual mediastinal lesion. It showed the presence of CD15 and CD30-positive RS-like cells (Fig. 1B and F) surrounded by various inflammatory cells, but not CD20 and CD79a-positive atypical large lymphoma cells (Fig. 1D). This result indicated a phenotypic change from GZL to cHL. Consolidative involved-field RT to the mediastinal lesion at a dose of $41.4 \mathrm{~Gy}$ was performed. However, after 2 months, PET/CT revealed a new FDG-uptake lesion in the lymph node in front of the liver. Then, CT-guided lymph node biopsy was performed, and this new lesion was found to have pathological features similar to those of CD15 and CD30-positive cHL, but not DLBCL. The patient underwent additional three cycles of ifosfamide, cisplatin, and etoposide (ICE) and sequentially received high-dose chemotherapy with ranimustine, etoposide, cytarabine, and melphalan, followed by ASCT. PET/CT scan prior ASCT revealed the residual lymph node in the mediastinum with FDG uptake (SUVmax $=3.53$, partial metabolic response), scored as 4 by Deauville 5-point scales (5-PS). However, the patient eventually achieved complete metabolic remission (CMR) with 5-PS score of 3 on PET/CT scan on day 90 after ASCT. Based on the clinical course showing treatment resistance, this patient was at extremely high risk of GZL recurrence. To decrease such a risk, the patient was treated with $\mathrm{BV}$ maintenance targeting CD30 expression in lymphoma cells. BV monotherapy maintenance $(1.8 \mathrm{mg} / \mathrm{kg}$ every 3 weeks) was initiated 3 months after ASCT. After 16 cycles of BV maintenance therapy, the patient was still on CMR and reached 5-PS of 1 on PET/CT scan on day 410 after BV initiation (Fig. 2B). Any adverse effects (AEs), such as peripheral neuropathy and neutropenia, were not observed during $\mathrm{BV}$ maintenance treatment.

\section{Discussion}

This report showed that BV maintenance after ASCT might be effective in a patient with R/R GZL. GZL is pathologically composed of cHL and primary mediastinal B-cell lymphoma (PMBL), and its component may change between cHL and PMBL during the clinical course (4). Our case showed the phenotypic change from GZL to cHL after DA-EPOCH-R and RT with sustaining CD30-positivity of lymphoma cells, therefore, $\mathrm{BV}$ targeting CD30 was considered to be a promising therapeutic agent in our case. However, GZL showed negative for CD30 in some cases $(2,4)$. Thus, re-biopsy of lymphoma lesion should be performed to confirm CD30 expression in lymphoma cells.

Previous reports showed that $\mathrm{BV}$ had a better prognostic impact on both R/R cHL and CD30-positive DLBCL. That is, the overall response rate (ORR) of R/R cHL and CD30-positive DLBCL were 75 and 44\%, respectively (6,7). In the AETHERA trial, the PFS and 5-year PFS of patients who received treatment with BV maintenance after ASCT for R/R cHL were 42.9 months and $59 \%$, respectively $(8,11)$. On the other hand, although a small number of cases, two patients with treatment-naïve CD30-positive GZL have been shown to benefit from combination of BV and chemotherapy (5). In other previous reports, BV was administered to 4 patients with R/R GZL, resulting in achieving complete remission in 2 patients and presenting resistant for BV

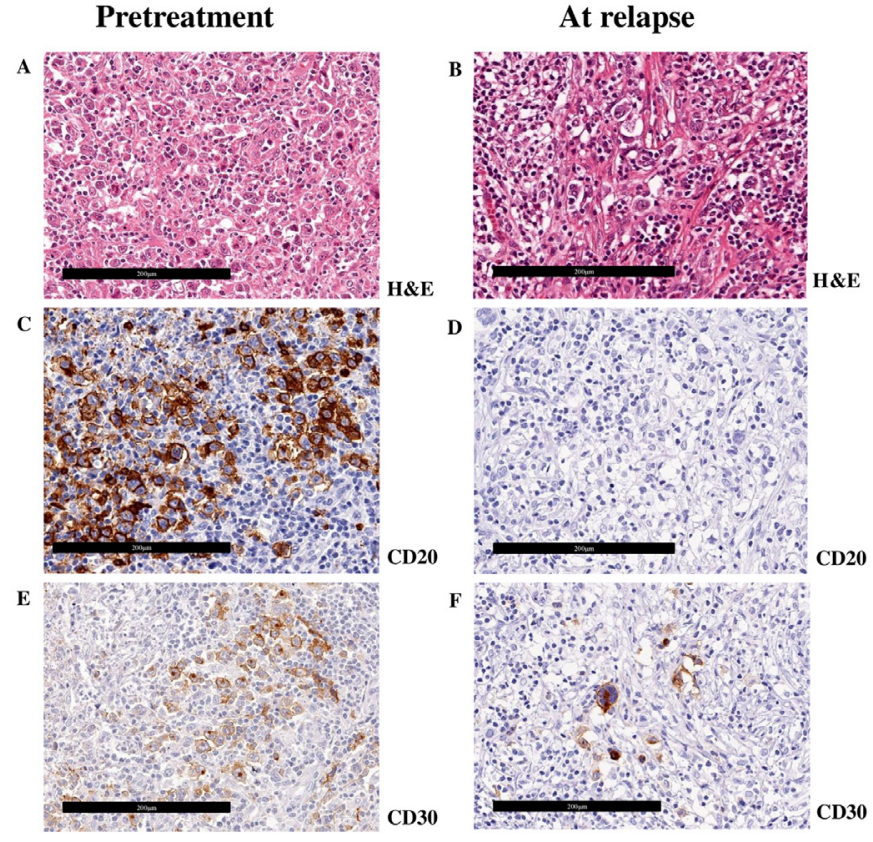

Figure 1. Pathological findings. Hematoxylin and Eosin Staining at (A) Pretreatment and (B) relapse revealed morphologic proliferation of atypical large lymphoma cells and the limited presence of polynuclear Reed-Sternberg-like cells surrounded by infiltrating inflammatory cells at pretreatment and relapse. These large lymphoma cells were positive for CD20 at (C) pretreatment; however, they became negative at (D) relapse. Lymphoma cells were positive for CD30 at both (E) pretreatment and (F) relapse. Scale bar, $200 \mu \mathrm{m}$.
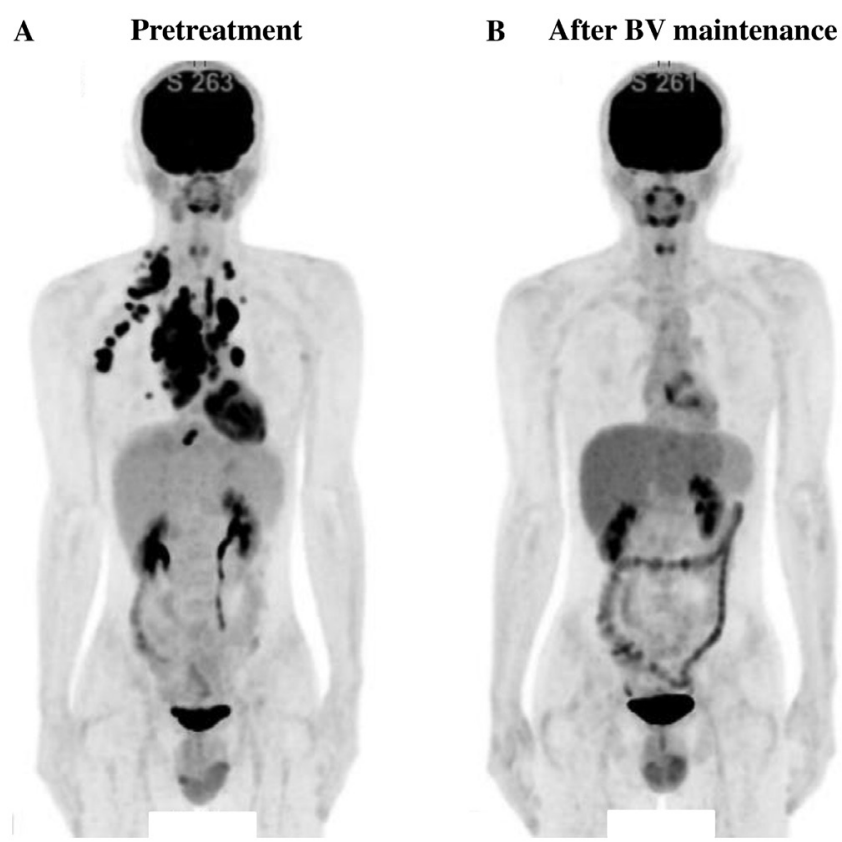

Figure 2. PET/CT. PET/CT images at pretreatment and after BV maintenance are presented. (A) Pretreatment PET/CT revealed hypermetabolic cervical, supraclavicular and anterior mediastinal masses (maximum standardized uptake value, 11.4). (B) After 16 cycles of BV maintenance, PET/CT demonstrated complete metabolic response. PET, Positron emission tomography; CT, computed tomography.

in another 2 patients $(9,10)$. Although these results showed the efficacy of BV for R/R GZL, this has been still unknown due to the rarity of this disease. Allogenic SCT is another salvage 
option for potentially achieving cure, however, it is concerned about transplant-related mortality and long-term toxicity. Our patient was at high risk of recurrence due to resistance to various chemotherapy (DA-EPOCH-R and ICE) and radiation, and the pathological diagnosis of residual disease prior ASCT showed a phenotypic change from GZL to cHL. Moreover, the prognosis of GZL is reported to be poorer than that of cHL or DLBCL (3). Considering of these points, our patient had received BV maintenance therapy after ASCT to lower the risk of recurrence based on the AETHERA study (11), and achieved long-term remission.

To date, our patient has maintained long-term remission after BV maintenance therapy. However, due to the history of resistance for treatment, he is still at risk of recurrence. At the time of recurrence after BV maintenance, the chemotherapy sensitivity of GZL is extremely low. Therefore, checkpoint inhibitors (CPIs) could be another therapeutic option. In patients with $\mathrm{R} / \mathrm{R}$ cHL who received nivolumab, the ORR and 6-months PFS were 66.3 and $76.9 \%$, respectively (12). In patients with R/R PMBL who received pembrolizumab, the ORR and 1-year PFS were $46 \%$ and $\sim 40 \%$, respectively (13). These data showed that CPIs might be an alternative therapeutic option for R/R GZL and could be a potential therapeutic option for our patient in future recurrence.

In summary, despite achieving CMR after ASCT, BV maintenance after ASCT was considered effective in a patient with GZL refractory to chemotherapy and RT, and severe AEs were not observed. Although a fraction of patients with $R / R$ GZL is often difficult to treat, the change in morphology and immunophenotype, including CD30 expression, at the time of recurrence should be confirmed. Hence, BV maintenance after ASCT could be an extremely effective therapeutic modality for transplant-eligible patients with CD30-positive GZL.

\section{Acknowledgements}

Not applicable.

\section{Funding}

No funding was received.

\section{Availability of data and materials}

The datasets used and/or analyzed during the current study are available from the corresponding author on reasonable request.

\section{Authors' contributions}

TT and JY wrote and edited the manuscript, provided patient care and reviewed the literature. NY, YMG and YM provided patient care and acquired the clinical data. KS analyzed and evaluated PET-CT images. MS and GI conducted pathological reviews. All authors read and approved the final manuscript. TT and JY confirm the authenticity of all the raw data.

\section{Ethics approval and consent to participate}

The present case study was approved by the Ethics Committee of the National Cancer Center Hospital East (approval no. 2018-416). The patient provided consent for inclusion in this study.

\section{Patient consent for publication}

The patient in this case provided consent to have this case published with removal of all identifying information to remain anonymous and retain privacy.

\section{Competing interests}

The authors declare that they have no competing interests.

\section{References}

1. Traverse-Glehen A,Pittaluga S, Gaulard P, Sorbara L, Alonso MA, Raffeld $\mathrm{M}$ and Jaffe ES: Mediastinal gray zone lymphoma: The missing link between classic Hodgkin's lymphoma and mediastinal large B-cell lymphoma. Am J Surg Pathol 29: 1411-1421, 2005.

2. Pilichowska M, Pittaluga S, Ferry JA, Hemminger J, Chang H, Kanakry JA, Sehn LH, Feldman T, Abramson JS, Kritharis A, et al: Clinicopathologic consensus study of gray zone lymphoma with features intermediate between DLBCL and classical HL. Blood Adv 1: 2600-2609, 2017.

3. Evens AM, Kanakry JA, Sehn LH, Kritharis A,Feldman T, Kroll A, Gascoyne RD, Abramson JS, Petrich AM, Herna dez-Ilizaliturri FJ, et al: Gray zone lymphoma with features intermediate between classical Hodgkin lymphoma and diffuse large B-cell lymphoma: Characteristics, outcomes, and prognostication among a large multicenter cohort. Am J Hematol 90: 778-783, 2015.

4. Kritharis A, Pilichowska M and Evens AM: How I manage patients with grey zone lymphoma. Br J Haematol 174: 345-350, 2016.

5. Svoboda J, Bair SM, Landsburg DJ, Nasta SD, Nagle SJ, Barta SK, Khan N, Filicko-O'Hara J, Gaballa S, Strelec L, et al: Brentuximab vedotin in combination with rituximab, cyclophosphamide, doxorubicin, and prednisone as frontline treatment for patients with CD30-positive B-cell lymphomas. Haematologica: May 15, 2020 (Epub ahead of print).

6. Younes A, Gopal AK, Smith SE, Ansell SM, Rosenblatt JD, Savage KJ, Ramchandren R, Bartlett NL, Cheson BD, de Vos S, et al: Results of a pivotal phase II study of brentuximab vedotin for patients with relapsed or refractory Hodgkin's lymphoma. J Clin Oncol 30: 2183-2189, 2012

7. Jacobsen ED, Sharman JP, Oki Y, Advani RH, Winter JN, Bello CM, Spitzer G, Palanca-Wessels MC, Kennedy DA, Levine P, et al: Brentuximab vedotin demonstrates objective responses in a phase 2 study of relapsed/refractory DLBCL with variable CD30 expression. Blood 125: 1394-1402, 2015.

8. Moskowitz CH, Nademanee A, Masszi T, Agura E, Holowiecki J, Abidi MH, Chen AI, Stiff P, Gianni AM, Carella A, et al: Brentuximab vedotin as consolidation therapy after autologous stem-cell transplantation in patients with Hodgkin's lymphoma at risk of relapse or progression (AETHERA): A randomised, double-blind, placebo-controlled, phase 3 trial. Lancet 385: 1853-1862, 2015.

9. Li J, Sim J, Leung AYH and Kwong YL: Favorable response of relapsed/refractory gray-zone lymphoma to brentuximab vedotin. Ann Hematol 97: 551-554, 2018.

10. Ebisawa K, Masamoto Y,Koya J, Shimura A, Shinozaki-Ushiku A, Toyama K, Nakazaki K and Kurokawa M: Long-term remission by brentuximab vedotin for non-mediastinal gray zone lymphoma refractory to autologous stem cell transplantation. Clin Lymphoma Myeloma Leuk 19: e602-e604, 2019.

11. Moskowitz CH, Walewski J, Nademanee A, Masszi T, Agura E, Holowiecki J, Abidi MH, Chen AI, Stiff P, Viviani S, et al: Five-year PFS from the AETHERA trial of brentuximab vedotin for Hodgkin lymphoma at high risk of progression or relapse. Blood 132: 2639-2642, 2018.

12. Younes A, Santoro A, Shipp M, Zinzani PL, Timmerman JM, Ansell S, Armand P, Fanale M, Ratanatharathorn V, Kuruvilla J, et al: Nivolumab for classical Hodgkin's lymphoma after failure of both autologous stem-cell transplantation and brentuximab vedotin: A multicentre, multicohort, single-arm phase 2 trial. Lancet Oncol 17: 1283-1294, 2016.

13. ArmandP, Rodig S, Melnichenko V, Thieblemont C, Bouabdallah K, Tumyan G, Özcan M, Portino S, Fogliatto L, Caballero MD, et al: Pembrolizumab in relapsed or refractory primary mediastinal large B-cell lymphoma. J Clin Oncol 37: 3291-3299, 2019. International (CC BY-NC-ND 4.0) License. 